\title{
Neurologists and the Numbers
}

Can. J. Neurol. Sci. 2005; 32: 399-400

The need for neurologists is growing. Neurology has undergone a major transformation over the past several decades from a largely diagnostic specialty to one with significant therapeutic involvement. There is an increasing need for neurological specialists to provide ongoing care for patients with chronic neurological disorders because of increasingly complex treatment regimens. Because neurological illness can be so devastating, a more sophisticated public is demanding more detailed evaluation of neurological symptoms including specialist consultation. This may well be appropriate. In a French study which evaluated the impact of a neurological consultation in the emergency department of a general hospital, it was found that neurological evaluation changed the diagnosis from that of the emergentologist in a major way in $52.5 \%$ of patients. ${ }^{1}$ The same study also demonstrated the high prevalence of neurological disorders in emergency departments. Neurologists were called to evaluate $14.7 \%$ of all patients admitted to the emergency department.

All these factors, combined with an aging population have resulted in a need for more neurologists. This trend is not new. In 1971 the National Institutes of Health in the United States (US) suggested that one pediatric neurologist was needed for every 100,000 children under the age of 15. A Canadian study concluded in 1991 that 2.5 to three pediatric neurologists per 100,000 children were needed. ${ }^{2}$ In keeping with these trends, over a 40 year time period, the number of postgraduate year two neurology residency positions in the United States increased by $338 \%$ from 130 in 1960 to 440 in $1998 .^{3}$

Neurologist / population ratios vary tremendously throughout the western world, and range from one neurologist per 8,117 population in Italy to one neurologist per 177,000 in the United Kingdom (UK). ${ }^{4}$ It seems noteworthy that many European countries, ranging from the Scandinavian countries (Norway, Sweden, and Denmark) to the Netherlands, Switzerland, France, and Greece have developed neurologist / population ratios of one neurologist to 20,000 to 40,000 population. ${ }^{4,5}$ The American Academy of Neurology has published figures ${ }^{6}$ which, when adjusted for the fact that only $80 \%$ of American neurologists belong to the Academy, indicate one neurologist per 23,500 population for the US. As reported in this issue of the Journal by Bailey et al, ${ }^{7}$ in 2002 there were 694 neurologists practicing in Canada, for a population ratio of one neurologist per 45,000 populaton. Clearly, in terms of neurologist numbers, Canada lags well behind the US and most Western European countries.

How many neurologists should there be in the future? This question likely cannot be answered in a useful way by central planners, and probably is best left, within reason, to the laws of supply and demand. While projections can be made, they by necessity depend on a number of assumptions, and these assumptions may not be borne out by reality in the future. Bailey et $a{ }^{7}{ }^{7}$ however, paint a disturbing picture for the future of Canadian Neurology. Unless neurology training programs increase their capacity, Canada is very likely to soon run seriously short of neurologists, and it may already be some distance down this road.

Twenty four neurologists per year are trained, according to their figures. At the same time, the neurology workforce is diminished every year by retirements. According to their survey, which was designed to capture data primarily on neurologists in adult neurology practice, $20 \%$ of current neurologists are currently over age 60, and appropriately, $20 \%$ of neurologists overall indicated an intention to retire in the next five years. Simple mathematics indicates, then, that we are already in a negative balance (the study was done in 2002), with 28 neurologists retiring each year, and only 24 coming in. This situation may deteriorate further very quickly. At the time when the neurologists currently over age 60 were trained, training programs were relatively small. Because of this, the cohort of neurologists currently over age 60 is also relatively small, and this influences the number of retirements. According to data from the survey by Bailey et $\mathrm{al}^{7}{ }^{7} 28 \%$ of Canadian neurologists currently are between the ages of 50 and 60, in contrast to the $20 \%$ of neurologists who are currently over age 60 . One could therefore expect the annual retirement rate to increase by approximately $50 \%$ as this larger cohort of neurologists reaches age 60 in the near future. In other words, unless training programs increase in size, we could in the near future have 24 neurologists entering the workforce annually from Canadian training programs and 42 leaving it for retirement. The world is of course larger than the boundaries of Canada. Canada should not have to depend, however, upon raiding other nations in order to meet its own neurological needs. At the same time, it has to be recognized that not all Canadian trainees stay in Canada for their entire careers.

Much has been said over the past several years about intentions to increase both medical school enrollment and the size of our specialty training programs, and some of this is happening. However, according to the Canadian Post MD Education Registry (CAPER), the number of Canadian citizens and permanent residents currently in Canadian adult neurology training programs at the R-5 ( fifth year resident level) is 23 . The corresponding figure for the R-4 level is 26 , and for the R-3 level is $22 .{ }^{8}$ Over the next three years, then, the output will on average be very close to the 24 per year cited by Bailey et al. ${ }^{7}$ There may be some reason for optimism, however, as the number of residents in Canadian adult neurology training programs at the $\mathrm{R}-2$ level is 29. These residents are at least three years from practice, and even if that number is maintained annually 
thereafter, it is still a long way from the projected 42 annual retirements noted above.

Considering that it takes at least five years to produce a neurologist, the time for action is now. Unless the number of neurologists trained each year increases substantially, Canadians with neurological disorders will be poorly served in the future. The UK offers an important lesson in this regard. A recent report endorsed by the UK Royal College of Physicians highlighted that with one neurologist per 177,000 population, the majority of patients admitted to hospital in the UK with acute neurological disease are not seen by a neurologist. ${ }^{5}$ To increase the number of UK neurologists from the current 358 to the 1400 considered necessary to provide UK hospitals with 24 hour neurologist coverage, it has been estimated that the number of neurologists trained each year would have to increase by more than 2.5 times and that it would take ten to 15 years to achieve the goal of 1400 neurologists. ${ }^{4,5}$ Clearly, once a nation gets behind in its specialty manpower, there is no quick fix.

In fact, it will be very difficult for Canada to train sufficient numbers of neurologists over the next several decades, even if training programs are increased significantly in size immediately. If patients with neurological disorders are to obtain good medical care in the future, a way must be found to make the limited Canadian neurologist supply go further.

An action which could be taken immediately to increase the efficiency of our current neurologists is the more widespread implementation of disease management teams. In his forward looking book, "From Chaos to Care", 9 David Lawrence, a chairman emeritus of Kaiser Permanente, makes a strong case that team based medicine is better for both patient and physician. According to the American Academy of Neurology, 28\% of American neurologists already work with physician extenders who help them in the care of patients. ${ }^{6}$ Corresponding Canadian figures for neurologists working with nurse practitioners, etc, are lacking, but may be significantly less. Research in a Canadian pediatric neurology clinic showed that, using a neurology nursing line, nurses were able to manage over half the calls received and needed to notify the physician in only $47 \%$ of cases. ${ }^{10}$ New technology is also able to amplify the ability of neurologists to provide service. A German study, which evaluated a stroke telemedicine service which included a videoconferencing system, concluded that this was a promising method to improve stroke care in rural areas. ${ }^{11}$ In fact, neurological consultation by teleconference resulted in a diagnosis other than stroke in $26 \%$ of patients diagnosed with stroke by the emergency room physician.

In conclusion, it is important that Canada act now to ensure that Canadians have adequate access to neurological services over the next several decades. Dual strategies are necessary. One of these, as advocated by Bailey et al, ${ }^{7}$ is to increase the size of neurology training programs. The other is to fund and develop more neurologist - non physician health professional teams to manage acute and chronic neurological disorders. The utilization of newer technologies including telemedicine should also be carefully investigated. Otherwise, as neurologist retirements outnumber new additions to the neurologist workforce, the ability of Canadian neurologists to serve the Canadian public will gradually diminish. As those leaving neurological practice outnumber new recruits year after year, the 694 neurologists currently in the system act as a buffer, but it will not take long for this imbalance to be felt. It is imperative that the Canadian Neurological Society continue to obtain good statistics with regard to the Canadian neurological workforce. Even more essential, it must work with the appropriate local and provincial authorities to implement solutions for the growing neurological manpower problem in Canada before it is too late.

W.J. Becker Calgary, Alberta

\section{REFERENCES}

1. Moulin T, Sablot D, Vidry E, et al. Impact of emergency room neurologists on patient management and outcome; Eur Neurol 2003; 50:207-214.

2. Ronen G, Meaney B. Pediatric Neurology services in Canada: demand versus supply; J Child Neurol 2003;18:180-184.

3. Bradley W: Neurology in the next two decades: report of the workforce task force of the American Academy of Neurology. Neurol 2000;54:787-789.

4. Butcher, J. Acutely ill neurological patients get raw deal in UK. The Lancet Neurology, 2002;1:4.

5. Warlow C, Humphrey P, Venables G. UK neurologists and the care of adults with acute neurological problems. Clinical Medicine 2002;2:436-439.

6. Swarztrauber K, Lawyer B. Neurologists 2000; American Academy of Neurology; St. Paul, MN, U.S.A., 2001.

7. Bailey P, Warren S, Buske L. Highlights of the 2002 Canadian Neurological Society (CNS) manpower survey; Can J Neurol Sci (in press).

8. Canadian Post MD Education Registry, Ottawa, Ontario, Canada (personal communication).

9. Lawrence D. From Chaos to Care - The Promise of Team Based Medicine. Cambridge: Perseus Publishing, 2002: MA, U.S.A.; 2002.

10. Letourneau M, MacGregor D, Dick P, et al. Use of a telephone nursing line in a pediatric neurology clinic: one approach to the shortage of subspecialists. Pediatrics, 2003; 112:1083-1087.

11. Wang D. Telemedicine. The solution to provide rural stroke coverage and the answer to the shortage of stroke neurologists and radiologists. Stroke 2003; 34:2951-2956. 